\title{
Tridimensional Spectroscopic Techniques: Conference Summary
}

\author{
J. Bland-Hawthorn \\ Anglo-Australian Observatory, P.O. Box 296, Epping, NSW 2121, \\ Australia
}

\section{Introduction}

Over the last four days, we have enjoyed a wide range of talks on developments in three dimensional spectroscopic techniques. The conference organizing committee are to be congratulated for the artful manner in which instrumental presentations were interleaved with talks on the scientific results from these instruments. The general thrust of most talks was to advance the versatility of traditional instruments either through the Jacquinot (throughput) advantage or through the multiplex advantage, or both. A number of groups have attempted to utilize the full aperture of scanning Fabry-Perot and Fourier Transform interferometers. Arguably, Fabry-Perot interferometers have a wider application at present, although imaging Fourier Transform devices appear to have finally arrived, at least in the near infrared.

We are fortunate to have had many of the innovators participate in this conference. The conference has paid particular homage to G. Courtès for his lifetime's work, particularly his advances in the development of focal reducers and enlargers, and multi-pupil array spectrometers. We also heard from $P$. Connes who has played a major role in the development of the Fourier Transform spectrometer (along with J.P. Maillard) and spherical etalons, to name a few. P.D. Atherton and N.K. Reay reported on progress and developments with the successful Queensgate etalon series currently in use by a large fraction of the conference participants.

There is not the space to provide a complete review of every talk and poster. In its place, I focus on those topics which formed the backbone of the conference: integral field spectroscopy, scanning Fourier Transform spectrometry (FTS), and imaging Fabry-Perot spectrometry. There is a brief discussion of integrated optical systems, followed by a short review of coherent and incoherent interference filters. I would also like to include some comments on detector developments since these play a major role in instrument design. We start with some highly provocative statements on the persistence of long-slit devices.

\section{Long-slit spectrometers}

A senior colleague, who would probably wish to remain anonymous (although he is the only person to have directed a major radio synthesis telescope in both hemispheres), delights in asking: "Why do you high energy people [infrared, optical, etc.] insist on using long slit spectrometers?" In certain respects, it is somewhat surprising that long-slit spectrographs maintain their stronghold as 
by far the most commonly used spectroscopic mode at every major observatory, and continue to be so on the new generation of large telescopes. The long-slit approach does not benefit from either the Jacquinot advantage or the Fellgett multiplex - strictly multichannel - advantage, with the rare exception of multislit spectrographs. Furthermore, when combining slits to form spatial maps, a common practice in galactic and extragalactic studies, there are at least three difficulties to overcome. The slits need accurate spatial registration; seeing disk variations cause losses at the slit jaws; and there may be significant atmospheric attenuation. The last two phenomena cannot be disentangled reliably in long-slit work.

A more damning comparison comes from considering how much information is actually used in the spectrogram when compared with other methods. The long-slit spectrogram is a highly sparse array. It is my suspicion that many astronomers would prefer not to be overwhelmed by a glut of data, arguing that a well-chosen slit orientation serves just as well. Some might even argue that the instrumental profile of a long-slit device is cleaner and better behaved than most other approaches. But to paraphrase a colleague, there are surely better ways to throw out $99 \%$ of the data than by plonking down a slit.

\section{Integral field spectrometers}

The Meudon group realized in the early 1980's the potential importance of multimode spectrographs (Vanderreist, Courtès \& Donas 1984). This lead to the development of SILFID which offers multi-fibre (MEDUSA mode) and multislit capabilities, in addition to packed fibre configuration for imaging (ARGUS mode). Dr. Courtès (1982) first initiated the concept of dividing up the focal plane of a conventional Cassegrain focus by using an array of hexagonal microlenses. Each of these is normally a few millimetres in size, and therefore, a focal enlarger is required to properly match each microlens to the seeing disk. The array of micropupils can now be dispersed, in a manner analogous to an objective prism observation of a star field, by a grism or a grating onto the detector. This approach has been used with great success by the TIGER system (Courtès et al 1988). There are no slit losses as such although, as for multi-fibre spectrographs, it is still important to match the seeing disk to the microlens apertures. The SILFID system (Vanderreist \& Lemonnier 1988) side-stepped the problem by offering three possible focal enlargements.

Judging from the scientific presentations, the integral field approach to imaging spectroscopy has clearly come of age. We have all witnessed the transition from paper tigers to an array of beautiful TIGER papers (see also Durret et al 1994; Rocca-Volmerange et al 1994). There were impressive talks on cooling flows, extended narrow-line regions in AGNs, star-forming activity in distant radio galaxies, and the stellar dynamics of nearby spiral galaxies. The [NII] $\lambda 6583$ observations of the energetic outflow in M51 were remarkably consistent with the HIFI observations of the same line (Cecil 1988). It would be highly informative to compare the photometric integrity of these independent data sets since the latter do not suffer any seeing losses.

It is instructive to compare the seminal paper by Kormendy (1988) on M31 with the new results from the Meudon group. The new data exemplify the ex- 
traordinary potential of going from one slit orientation to full two dimensional absorption line spectroscopy. Wilkinson et al. (1986) attempted something similar with ASPECT (Clark et al 1984), a scanning long-slit mode at the AngloAustralian $3.9 \mathrm{~m}$ telescope (AAT). The important differences between the ASPECT and TIGER approaches were elucidated in the previous section. TIGER does not solve the more general problem of spatially imaged spectroscopy for objects with a fast decline in surface brightness. It is interesting to note that the underlying potential in elliptical galaxies is changing fastest where the surface brightness declines most rapidly (Binney \& Tremaine 1987). Therefore, we do not need the same spatial sampling over the full field. The study of elliptical galaxies would surely be revolutionized if it was possible to collect flux within elliptic annular apertures whose thickness, semi-major axes and orientations were adjustable parameters. Do microlens arrays really need to be held to a fixed contiguous pattern?

From an instrumental perspective, current limitations with the TIGER approach appear to be the number of microlenses $(400)$ and the use of circular lenses embedded in hexagons with the attendant loss in area (10\%). However, the next generation integral field spectrograph at the CFHT will quadruple the number of microlenses, and these will comprise truly hexagonal optics. The geometric microlens pattern leads to an inefficient use of the detector area. An entrance filter is used to ensure that spectra do not overlap in the dispersion direction. Furthermore, the disperser is rotated through a small angle to avoid overlap perpendicular to the dispersion. A solution to the inefficient use of detector area was touched on by V. Afanasiev. Their TIGER-like spectrograph at the $6 \mathrm{~m}$ Caucasus telescope uses 225 microlenses, each of which images the pupil onto the front face of an optical fibre. Once inside the fibre, it now becomes straightforward to pack the data onto the detector by lining up the fibres along the entrance slit. This removes the need for a blocking filter also. Seifert (see also Felenbok et al 1994) showed how a similar approach is to be adopted by the FUEGOS system under construction for the ESO Very Large Telescope (VLT).

Proper sky subtraction appears to be a limitation with the TIGER and ARGUS approaches. This recalls the long-standing issue of whether multi-slit spectrographs, with the adjacent sky coverage, are a better approach than multifibre spectrographs which are less susceptible to seeing losses. In practice, it largely depends on the application. $H$. Ohtani has addressed this issue in the multi-mode spectrograph for the Okayama $1.88 \mathrm{~m}$ telescope (but see $\$ 9$ ). A dualchannel preoptics system is used to observe the sky simultaneously by directing the light into a small fraction of the microlenses (Ohtani et al 1994).

\section{Scanning Fourier Transform interferometers}

The two most common applications of Fourier Transform interferometry divide either the wavefront (lamellar gratings; see Meaburn [1976]) or the wave amplitude (Michelson interferometer). It is often forgotten that the FTS design seen in most texts (e.g. Hecht 1990) throws away $50 \%$ of the light. It is possible to recover this light but the layout is somewhat involved (Connes \& Michel 1975; Larson \& Fink 1975; Hall et al 1979). While these instruments have promised much for decades (Connes 1970; Maillard 1973), successful imaging spectro- 
graphs have been a long time coming ${ }^{1}$ (Maillard \& Simons 1992; Simons et al 1994). One only has to recall the words of Bell (1972): "Considering the sorting ability of the four instruments in order, the prism, the grating, the Fabry-Perot interferometer, and Michelson interferometer, the number of beams decreases monotonically, but the energy throughput increases, thereby implying the fewer the number of beams the greater the energy. The function of all these instruments ultimately depends on interference, and the phenomenon of interference requires at least two beams; therefore, two-beam interferometers have ultimate throughput or efficiency." How far has this been realized?

Various groups presented impressive results for several bright sources including Venus, Jupiter, the planetary nebula NGC 7027, and the proto-planetary nebula CRL 2688. For these objects, the advantage over the Fabry-Perot interferometer is clear. From the FTS data cube, it is possible to extract information on a wide range of emission and absorption lines. In the same exposure time, the Fabry-Perot would be forced to scan sequentially in the vicinity of each line, thereby bypassing most of the intervening continuum. However, these are very bright infrared sources. An interesting comparison has recently become possible. V.M. Meadows and D.A. Allen of the Anglo-Australian Observatory have obtained spectacular three-dimensional data with IRIS (Allen et al 1993) for both Jupiter $^{2}$ and Venus. Much like the ASPECT approach, the slit was scanned across the face of the planets, albeit at a much faster scan rate.

An important comparison of the FTS and the scanning Fabry-Perot has been made by Geballe (1982). In the $1-2.3 \mu \mathrm{m}$ window, $\mathrm{OH}$ lines account for almost all of the background (Oliva \& Origlia 1992; Ramsay et al 1992). This has given rise to a new generation of $\mathrm{OH}$-suppressing spectrographs (Maihara et al 1994). With an FTS, the airglow lines greatly increase the background at the detector. This requires that we isolate the spectral region of interest with a blocking filter. The advantage of the Fabry-Perot is that one can specify precisely the wavelength interval to be scanned, thereby avoiding the strong $\mathrm{OH}$ lines.

It seems plausible that the FTS is the ideal device for imaging extended absorption-line systems. The instrumental profile is easily modified by convolution so as to ensure a compact Fourier transform which is essential for the deconvolution process. In some respects, Lorentzian spectrometers (e.g. FabryPerots) are seriously limited by the wing power which is not aided by going to higher finesse (Bland-Hawthorn \& Jones 1994). To return to the issue of spheroidal galaxies raised in the previous section, the data can be binned in logarithmic intervals to combat the rapid decline in the surface brightness. The problem with using either device is that the required rapid scanning runs up against the background-noise limit of infrared detectors. Presently, it is difficult to maintain low read noise levels with fast read cycles, since the low noise levels are achieved with multiple read outs.

The primary technical challenge with FTS devices is the stringent rigidity (path equalization) requirements. Small amounts of mechanical flexure can be

${ }^{1}$ J.P. Maillard points out that irnaging Fourier Transform devices in the infrared could not have appeared much earlier than 1991 which saw the introduction of the $\mathrm{HgCdTe}_{\mathrm{g}}$ arrays.

${ }^{2}$ This principle was used to great effect during the recent SL9 cometary collision with Jupiter. 
problematic although it may be possible to remove these effects in software (Simons et al 1994). The moving mirror control can be difficult for the highest resolving powers $\left(>10^{5}\right)$. The standard approach is to use a servo system built around a stabilised laser. An ideal system would have a groove-less mirror spacing control since periodic defects generate spurious frequencies in the observed signal. To combat this, A.P. Thorne and collaborators at Imperial College, London have succeeded with a hydraulic piston arrangement. Other technical challenges include phase shifts introduced into the interferogram either by the electronics or the beam splitter dispersion.

After a fairly lengthy critique, it is important to remember that the FTS offers extraordinary versatility, in particular, the freedom to choose the spectral resolution over an (almost) arbitrary spectral range. The FTS realizes all of its advantages at high spectral resolution over a wide spectral band. Moreover, J.P. Maillard demonstrated the robustness of the FTS to scattered light. This signal is usually not modulated by the optics and is imaged at the detector as an additional d.c. level in the interferogram. This extra signal is easily masked from the transform as it is confined to the lowest few wavenumbers.

\section{Scanning Fabry-Perot interferometers}

We have seen presentations from roughly half of the world's astronomical FabryPerot systems. Data was presented from Fabry-Perots operating in AAT, Arizona (Ohio), Chile, CFHT, La Palma, UKIRT and Wisconsin with the most extensive presentations coming from the Marseilles and Hawaii groups. While most groups use high-performance detectors and Queensgate etalons, it is somewhat surprising that few groups appear to be doing spectrophotometry. This issue has been addressed elsewhere in the proceedings. The case was made for expending a lot of effort in tracking down and exorcising the various ghost families. The impact of ghost reflections, and scattered light in general, was evident in a number of the presentations. When these anomalies are removed, the Fabry-Perot is capable of high quality spectrophotometry on a par with, if not better than, what is possible with narrow-band imaging and long-slit techniques. This being said, there are many compromises in the design, construction and application of Fabry-Perot interferometers.

A major technical advance for Fabry-Perot interferometers has been the development of multi-layer dielectric coatings with their low absorptance and high reflectivity compared to metal coatings (MacLeod 1969). However, the much higher performance has emphasized the surface defects of the substrates. Queensgate Instruments are able to polish etalon plates to a flatness of $\lambda / 200$ which, to quote P.D. Atherton, 'is like flattening a football field to $10 \mu \mathrm{m}$ '. Fizeau interferometry is used to check the plate flatness and this is about the limit of useful detectability (Malacara 1988).

It is to be remembered that the effective finesse $\mathcal{N}_{\mathrm{E}}$ of the Fabry-Perot is the net effect of the reflective, aperture and defect finesse (Atherton et al 1981). To achieve $\mathcal{N}_{E}=100$ requires the plates to be flat to at least $\lambda / 200$. 
However, it is difficult to deposit coatings with this level of flatness. ${ }^{3}$ E. Pelletier discussed ion bombardment as a more accurate way of compressing the coating layers during evaporation. While this looks highly promising, physical stresses within the dielectrics appear to warp the plates, independent of surface area, due the increased surface tension (Ennos 1966). Surface defects cause light to be scattered out of the beam. Fig. 1 illustrates the drastic loss of efficiency as a function of finesse. The peak to wing contrast of the Airy profile increases quadratically with $\mathcal{N}$ (Bland \& Tully 1989). Thus, an improved instrumental profile is a compromise with a loss of efficiency. To test the degree of light scattering, compare the monochromatic response of the Fabry-Perot with the theoretical response. A lower than predicted Airy peak to trough contrast could well be due to the light scattered by plate defects.

An important similarity of Fabry-Perot and Fourier Transform interferometers is the broad wavelength restriction. On the one hand, the beam splitter for the FTS can only be optimized for a limited spectral range. Currently, a similar problem arises with etalon coatings. Queensgate Instruments are able to achieve an arbitrarily high reflective finesse but only over a narrow wavelength interval $(\sim 0.2 \lambda)$. Longer wavelength intervals require many more dielectric layers and these have been optimized only for relatively low $(<30)$ finesse values.

The scientific presentations have served to underline the versatility of the Fabry-Perot. There were memorable talks on high velocity outflows from AGNs and starbursts, on star formation regions, supernova remnants and planetary nebulae. There were extensive surveys of the Milky Way and the Magellanic Clouds. In addition, there were deep observations of extended gas in elliptical galaxies and gaseous 'haloes' in high-redshift radio galaxies. Solar system studies included detections of comets and the Io torus which were all the more remarkable for the additional temporal information. In some cases, the etalons were used at low finesse simply for accurate narrow-band imaging. More often, the contiguous data frames were stacked to form a data cube. A third and largely unexplored approach is to exploit the Jacquinot advantage to the full by observing diffuse sources that fill the field; a single spectrum is obtained after azimuthal binning (annular summing). The Wisconsin group have used this to good effect to observe the warm ionized medium in the Milky Way (q.v. Reynolds et al 1990).

In passing, the flux transmitted by the etalon is only a small fraction $\left(\mathcal{N}^{-1}\right)$ of the reflected light. Are there possible applications for the reflected light? Note from Fig. 1 that, at relatively low finesse, very little of the light is lost to scattering. One possible application is for suppressing $\mathrm{OH}$ lines in near-infrared imaging. Within a small tolerance, the $\mathrm{OH}$ band heads are highly periodic. The idea would be to take the power spectrum of the predicted $\mathrm{OH}$ spectrum within some window (Traub \& Stier 1976; Abrams et al 1994) and match the free spectral range and the finesse to the power spectrum feature(s). A possible restriction is the small Jacquinot spot size at a sufficiently high resolving power to separate the OH lines.

\footnotetext{
${ }^{2}$ For reasons that are not understood, and therefore not repeatable, Queensgate Instruments have on occasion measured $\lambda / 200$ flatness after the costings have been deposited.
} 


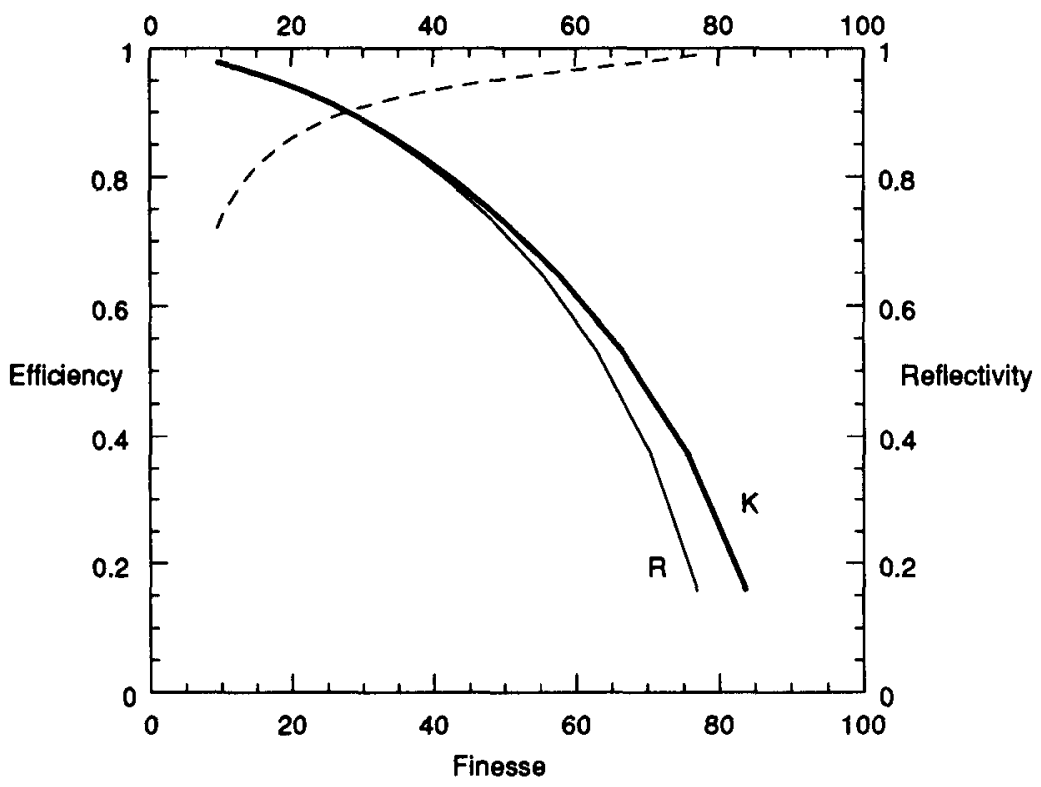

Figure 1. A plot of efficiency vs. effective finesse for $\mathbf{R}$ and $\mathbf{K}$ band Queensgate etalons. The $R$ band calculation is for a wavelength of $6700 \AA$ and a plate flatness of $\lambda / 150$ (after coating) measured at $6330 \AA$. The $\mathrm{K}$ band etalon is for a wavelength of $2.2 \mu \mathrm{m}$ and a plate flatness of $\lambda / 50$. The curves decline less rapidly if a higher plate flatness is achieved. We have included losses of $0.2 \%$ due to coating absorption and scattering; these tend to have more effect at higher finesse. The dashed curve shows how the finesse depends on the coating reflectivity. 


\section{Integrated optical systems}

An important trend appears to be the use of integrated optical systems. Exam. ples we have seen at this conference are the CFHT Optically Adaptive System for Imaging Spectroscopy (OASIS ${ }^{4}$ ) and the Okayama multi-mode spectrographic and imaging system. The median seeing at the CFHT is around $0.75^{\prime \prime}$ and somewhat better with fast tip-tilt corrections. The PUEO Adaptive Optic Bonnette system (Arsenault 1994) is soon to come on line and is designed for easy use with all Cassegrain instruments. The OASIS system has half a dozen modes including TIGER, ARGUS, PYTHEAS, imaging, long-slit and Fabry-Perot modes. The ARGUS mode is similar to the TIGER mode (see §3), albeit with a smaller field of view and a larger spectral range. PYTHEAS (le Coarer et al 1993) is an adaption of TIGER that achieves echelle resolutions at the Cassegrain focus with the aid of an etalon. An important limitation is that the etalon needs to be scanned through $2 \mathcal{N}$ steps and the spectra need to be reconstructed in software from a series of combs on the detector.

The multi-mode spectrograph for the Okayama $1.88 \mathrm{~m}$ telescope has been designed with the prospect of using on the SUBARU $8 \mathrm{~m}$ telescope at Mauna Kea Observatory. This system offers imaging and long-slit spectrographic modes, a Fabry-Perot system and a microlens array (Ohtani et al 1993). Both integrated systems have been designed with exceptionally small pixel scales to fully exploit the diffraction-limited images. The beauty of integrated optical systems is that all facilities are easily accessible on the same Cassegrain focal bench and, when required, adaptive optics can in principle be slid into position in a matter of minutes.

\section{Incoherent and coherent interference filters}

Spectrometers and interference filters, while both used as monochromators, have an important difference (Bland-Hawthorn \& Jones 1994). For the spectrometer, the ideal instrumental profile is the triangular function in contrast to the interference filter where the ideal response is the top-hat function (rectangular function, Butterworth profile). In the former case, the profile is convolved with the observed spectrum: the triangular function has smaller side lobes than the top-hat function in the Fourier domain. In the latter case, the profile is simply multiplied with the observed spectrum.

Modern interference filters are, of course, Fabry-Perot interferometers constructed with thin films (MacLeod 1969). A single cavity filter comprises alternate layers of high and low refractive index material applied to a substrate. The filter response can be made more 'top hat' in shape by sandwiching cavities in series (multi-cavity filter). Off-band rejection is achieved by colour-absorbing glasses or dyes at the short wavelength cut-off, and metallic or dielectric coatings at the long wavelength cut-off. The Lorentzian (strictly Airy) nature of the basic Fabry-Perot filter is a fundamental limitation. There have been alternative approaches to changing the shape including multi-mirror systems where one or

${ }^{1}$ This is not to be confused with the Okayama Astrophysical System for Infrared imaging and Spectroscopy (Iye 1994). 
more partially reflecting mirrors are used in series with the basic Fabry-Perot cavity (Gunning 1982; van de Stadt \& Muller 1985).

In his talk, J. Caplan suggested ways to deconvolve the effect of the Airy function by rapid sampling. During the subsequent discussion, it was suggested that the Airy profile could be modified by moving the plates rapidly during a single exposure in such a way that the time spent at different gap spacings is given by the coefficients of the expansion of an ideal function. The inherent limitation with this approach is that the transmitted flux is not the band-pass of the newly generated square profile, but the effective band-width of the Airy profile.

F. Roesler reviewed recent developments at Wisconsin. The PEPSIOS system (Mack et al 1963) uses three etalons in series. This is an incoherent superposition of etalons in that reflected beams among individual etalons are suppressed. McNutt (1965) has determined the general case of $m$ etalons in series. The etalons are scanned simultaneously to achieve spectral resolving powers of order $10^{6}$. It would be interesting to compare the performance with standard echelle instruments and with the PYTHEAS approach.

\section{Have astronomical detectors reached their performance limits?}

The world population seems to divide into three main groups: pessimists, optimists and incurable optimists. Some of the techniques that we have heard about in the last few days have been on the drawing boards for more than a decade. Since, for a given telescope, many or most instruments are designed backwards from the available detectors, it is clear that certain groups took faith in the prospect of significant developments in the performance and size of CCDs. We are all aware that infrared detectors are evolving rapidly in their performance, particularly in the $1-3 \mu \mathrm{m}$ region, to the point that modern infrared spectrographs are not so far behind optical instruments. However, one often reads in review papers that 'optical detectors have finally reached their theoretical performance limits.' Is that true?

One could argue that CCDs still have a way to go before we are in a position to use them as photon counting arrays. Only then will CCDs effectively close the books on competing technologies. It would seem that $\mathrm{V}$ to $\mathrm{R}$ band quantum efficiencies near $80 \%$ come fairly close to the theoretical limit of CCDs. However, there is a variety of other factors to consider including dynamic range, read noise limits, read out times, detector formats and pixel sizes, each of which are addressed below.

The current read noise levels of CCDs at most national facilities are anywhere between 2 and $5 \mathrm{e}^{-}$. With the aid of non-destructive readout amplifiers ('skipper CCDs'), this can be pushed to significantly below the level of $1 \mathrm{e}^{-}$, although primarily for point sources rather than extended, low-level sources (Janesick \& Elliot 1991). Sub-electron imaging has already been achieved by Tyson (private communication) on $10^{6} \mathrm{e}^{-}$well devices. At infrared wavelengths, Fowler and Gatley (1990) have used related methods to drastically reduce the read noise of SBRC arrays.

Until recently, the smallest detector pixels were $15 \mu \mathrm{m}$. However, Loral have taken one of their $2048 \times 2048$ chips and produced a detector with a $4096 \times 4096$ 
format and $7.5 \mu \mathrm{m}$ pixels. At least half a dozen groups have achieved these format dimensions by edge-butting chips together (Luppino et al 1994, Table 3). To quote yet another colleague, who really does wish to remain anonymous (I.R. Parry), 'the cost of detector real estate is still falling'. Orbit, Inc. have recently announced a $5192 \times 5192$ CCD array with $12 \mu \mathrm{m}$ pixels for US\$75,000; Dalsa, Inc. are offering a $5120 \times 5120$ array also with $12 \mu \mathrm{m}$ pixels for only US\$50,000. The current problem with using large detector formats are the prohibitively long read-out times. But this shows signs of being resolved ultimately as we now discuss.

A crucial step in achieving a performance close to photon counting arrays is already being realized at the Jet Propulsion Laboratory. The Spatial Interferometry Group are developing a $32 \times 64 \mathrm{CCD}$ with a frame rate of $1.5 \mathrm{KHz}$ at a cost of only $\$ 10,000$. The Adaptive Optics CCD (ACCD) will have characteristics that are close to contemporary optical CCDs: $30 \mu \mathrm{m}$ pixel size, $1.5 \mathrm{e}^{-}$read noise, $80 \%$ maximum quantum efficiency, albeit with a somewhat diminished full well capacity at $10^{4} \mathrm{e}^{-}$. The ACCD will have an output port on each of the columns and will use skipper on-chip amplifiers. Larger fast read-out arrays are being produced by Lawrence Livermore National Laboratory and Loral Fairchild, Inc.

Finally, the linear dynamic range of CCDs is presently at the level of $1: 10^{5}$ (Tyson 1990) although this limitation is set by the amplifiers. From a theoretical perspective, Eppeldauer \& Hardis (1991) have shown that silicon has a dynamic range of at least $1: 10^{14}$. Budde (1979) has demonstrated more than eight orders of magnitude dynamic range in the laboratory. One can only wonder at the possibilities raised by superconducting tunnel junction and charge injection devices. It is to be expected that future spectrograph design will ultimately benefit from the developments described here.

\section{Special appeal}

There was little discussion about the various shortcomings of each instrument. Detailed comparisons between instruments would have helped here. As a general rule, optical and infrared astronomers do not go to the same lengths to understand their noise sources and degradations as, say, radio astronomers. All instruments are susceptible at some level to scattered light. This is rarely discussed in the context of spectrographs but is surely a bigger problem than is given credit for, as multi-fibre spectroscopists are now discovering (Wyse \& Gilmore 1992). The issue of ghost reflections in Fabry-Perot interferometers is discussed elsewhere in these proceedings; a related discussion for Fourier Transform devices would also be useful. Just how well can you flatfield and sky-subtract observations with microlens arrays? Ohtani et al (1994) obtain adjacent sky coverage with their microlens array but a different optical path is used (\$3). As CCDs have grown, the images at the slit ends have become increasingly distorted and defocussed. Seifert mentioned that there will be non-negligible point spread variations across the FUEGOS detector field which will certainly complicate sky subtraction. 


\section{Communing with nature}

Astronomers and space scientists clearly love to use acronyms. These range from the wonderfully contrived (e.g. $\mathrm{PUEO}^{5}$ ) to the blatantly contravenous (e.g. HIFI). Heck (1992) has compiled more than 55,000 of these in a weighty compendium that makes for good bedtime reading. As an historical aside, Dr. Atherton observes that the use of meaningful acronyms for instrumentation was largely unheard of fifteen years ago. It is interesting to note that, in the mould of Mayan astronomers, we continue to commune with nature: CIGALE (ciccada) and ARGUS; PALILA and PUEO (Hawaiian birds); BEAR; PUMA and TIGER; and so on. Both fish and reptiles are seriously underrepresented at present. On a more elemental plane, we heard talks on the WINDII, FUEGOS and OASIS; a pedological theme (e.g. Long-slit Optical Array Matrix, or Complex Lens ArraY system) would complete the set.

\section{Conclusions}

In the present era of 8-10 $\mathrm{m}$ telescopes under construction, the conference was a timely reminder there are other options than simply scaling up yesterday's technology. I would like to end on an upbeat note for tridimensional spectroscopic techniques. The diversity of applications to astronomical objects exemplified by the conference is impressive. We heard talks on geocoronal $\mathrm{H} \alpha$, the EarthMoon system, Venus and Mars, the Jupiter-Io system, the Sun, comets, nearby stars, the warm ionized gas in the Galaxy, planetary nebulae and HII regions, Herbig-Haro objects, nearby and active galaxies, quasars and high redshift galaxies. It is clear that 'tridimensional spectrographs' have already claimed much of the traditional domain of long-slit spectroscopy. However, some would argue that few (if any?) of the instruments can be considered truly general at present and that most are best suited to less than a dozen astronomical projects. For now, it is unlikely that any particular technique is being squeezed out by advances in other fields. I have tried to highlight the limitations and advantages of the various approaches to tridimensional spectroscopy. One anticipates that a number of these techniques will ultimately find a footing on the $8-10 \mathrm{~m}$ class telescopes, operating at both optical and infrared wavelengths.

Let me close with one final remark. France is arguably the most innovative nation when it comes to optical design in astronomy. It is clear that, at least from a Marsellaise perspective, this is partly the legacy of Charles Fabry and Alfred Perot. We heard at the end of the first day of the extraordinary legacy left behind by these great pioneers: the first detailed spectroscopic studies of the ozone layer and the Orion nebula, detection of gravitational redshift in Solar spectral lines, verification of the Doppler-Fizeau principle, and most crucially, the giant leap that became possible in defining internationally accepted measurement standards. One notes that the anniversary for the invention of the device that made this possible is only four or five years away. May I be so bold as to suggest that Marseilles would once again provide the perfect setting for this important celebration?

\footnotetext{
${ }^{5}$ Probing the Universe with Enhanced Optica
} 
Acknowledgments. I am indebted to Lady Anne Thorne, Christian Vanderreist, Ian Parry and Gerald Cecil for their insights, and particularly to JeanPierre Maillard for his detailed comments. I am grateful to Peter Fellgett, FRS for emerging from the somnolent bliss of his Devonshire cottage and sharing his historical perspective. I would like to extend thanks to the staff of Queensgate Instruments, Ltd. for their friendship and tireless assistance, particularly Paul Atherton, Ken Reay and Chris Pietraszewski (who assisted with Fig. 1). Finally, I would like to pay special tribute to D.A. Allen whose untimely death is felt deeply by the Australian astronomical community. In addition to a (short) lifetime of outstanding research, David made wide ranging contributions to the development of both optical and infrared instrumentation.

\section{References}

Abrams, M.C. et al 1994, ApJS, 93, 351

Allen, D.A. et al 1993, PASA, 10, 298

Arsenault, R. et al 1994, preprint

Atherton, P.D. et al 1981, Opt. Eng. 20, 806

Bell, R.J. 1972, Introductory Fourier Spectroscopy, Academic Press

Binney, J. \& Tremaine, S.D. 1987, Galactic Dynamics, Princeton

Bland, J. \& Tully, R.B. 1989, AJ, 98, 723

Bland-Hawthorn, J. \& Jones, A.W. 1994, preprint

Budde, W. 1979, Appl. Opt., 18, 1555

Cecil, G.N. 1988, ApJ, 329, 38

Clark, D. et al 1984, QJRAS, 25, 114

Connes, P. 1970, ARA\&A, 8, 209

Connes, P. \& Michel, G. 1975, Appl. Opt., 14, 2067

Courtès, G. 1982, IAU Coll. 67, 123

Courtès, G. et al 1988, In Instrumentation for Ground-based Optical Astronomy, IXth Santa Cruz workshop, p. 266.

Durret, F. et al 1994, A\&Ap, in press

Ennos, A.E. 1966, Appl. Opt., 5, 51

Eppeldauer, G. \& Hardis, J.E. 1991, Appl. Opt., 30, 3091

Felenbok, P. et al 1994, Instrumentation in Astronomy VIII, SPIE vol. 2198, p. 115

Fowler, A.M. \& Gatley, I. 1990, ApJ, 353, L33

Geballe, T.R. 1982, Proc. of the 2nd ESO Infrared Workshop, Garching

Gunning, W. 1982, Appl. Opt. 21, 3129

Heck, A. 1992, StarBriefs, Observatoire Astronomique, Strasbourg, France

le Coarer, E. et al 1993, A\&A, in press

Luppino, G.A. et al 1994, In Instrumentation in Astronomy VIII, SPIE vol. 2198, p. 810 
Hall, D.N.B. et al 1979, In Instrumentation in Astronomy III, SPIE vol. 172, 121

Hecht, E. 1990, Optics, Addison-Wesley, Massachussetts, 2nd ed., p. 383.

Iye, M. 1994, In Instrumentation in Astronomy VIII, SPIE vol. 2198, p. 153

Janesick, J. \& Elliot, T. 1991, In History \& Advancement of Large Area Array Scientific CCD Imagers, PASP Conf. Series, Tucson

Kormendy, J. 1988, ApJ, 325, 128

Larson, H.P. \& Fink, U. 1975, Appl. Opt., 14, 2085

Mack, J.E. et al 1963, Appl. Opt., 2, 873

MacLeod, H.A. 1969, Thin Film Optical Filters, Adam Hilger, London

Maihara, T. et al 1993, PASP, 105, 940

Maillard, J.P. 1973, PhD, Universite du Paris-Sud, Centre d'Orsay

Maillard, J.P. \& Simons, D.A. 1992, In ESO Workshop on Solar Physics \& Astrophysics at Interferometric Resolution, Paris, p. 205

Malacara, D. 1988, Meth. Expt. Phys., 26, chap. 1

McNutt, D.P. 1965, JOSA, 55, 288

Meaburn, J. 1976, Detection and Spectrophotometry of Faint Light, Reidel

Ohtani, H. et al 1994, In Instrumentation in Astronomy VIII, SPIE vol. 2198, p. 229

Oliva, E. \& Origlia, L. 1992, A\&A, 254, 466

Ramsay, S.K., Mountain, M. \& Geballe, T.R. 1992, MNRAS, 259, 751

Reynolds, R.J. et al 1990, In Instrumentation in Astronomy VII, SPIE, vol. 1235

Rocca-Volmerange, B. et al 1994, A\&Ap, in press

Simons, D.A. et al 1994, In Instrumentation in Astronomy VIII, SPIE, vol. 2198, p. 185

Tokunaga, A. 1976, PhD, State Univ. of New York, Stony Brook

Traub, W.A. \& Stier, M.T. 1976, Appl. Opt., 15, 364

Tyson, J.A. 1986, Appl. Opt., 3, 2131

Vanderreist, Ch., Courtès \& Donas, J. 1984, J. Opt., 15, 237

Vanderreist, Ch. \& Lemonnier, J.-P. 1988, In Instrumentation for Ground-based Optical Astronomy,, IXth Santa Cruz workshop, p. 304.

van der Stadt, H. \& Muller, J.M. 1985, JOSA, 2, 1363

Wilkinson, A. et al 1986, MNRAS, 218, 297

Wyse, R.F.G. \& Gilmore, G. 1992, MNRAS, 257, 1 